\title{
Revealing mechanical and structural properties of molecules on surface by high-resolution atomic force microscopy
}

\author{
Shigeki Kawai ${ }^{1,2}$ \\ The state-of-the-art atomic force microscopy allows us to observe inner structures of single molecules. Such observation is quite \\ beneficial to study single and self-assembled molecules on surface as well as on-surface chemical reaction. This focus review \\ describes recent technical improvement and achievements in the field of atomic force microscopy.
}

Polymer Journal (2017) 49, 3-11; doi:10.1038/pj.2016.106; published online 19 October 2016

\section{INTRODUCTION}

Ever since scanning tunneling microscopy (STM) was invented, ${ }^{1}$ we can study surfaces with sub-nanometer resolution. In this technique, although a bias voltage between tip and sample is applied (typically $< \pm 5 \mathrm{~V}$ ), the detected tunneling current is used to control the tipsample distance. As the distance dependence of the tunneling current is very strong, the surface can readily be imaged with high resolution in real-space. As molecules on surfaces are one of the most attractive topics, a number of studies, such as crystallization, manipulation and chemical reaction, have so far been reported. ${ }^{2}$ Although this technique is reliable, the interpretation of the observed image is not straightforward as the contrast often relates to the electronic structure of the molecules. ${ }^{3}$ In the case of molecules, complex molecular orbitals often obscure the structural information. For this reason, the density functional theory calculation is required to gain a detailed insight.

Recently, the resolution of atomic force microscopy (AFM) improved drastically by using a functionalized tip and hence the inner structures of molecules can be resolved directly via the frequency shift. ${ }^{4}$ In such measurements, a metal tip is functionalized by a CO molecule, picked up from the surface. The so-called CO functionalized tip can detect the Pauli repulsive interaction, which has a stronger $Z$ distance dependence than that in the attractive force, and so that the resolution can be improved. ${ }^{5}$ Furthermore, the tilt effect of the CO molecule on the tip apex by the interaction force induces a sharp contrast of the bond. ${ }^{6}$ Although the tip preparation is still rather challenging, this technique becomes established. The ability of the direct observation is very beneficial to study chemistry on surface, ${ }^{7}$ as the structure of the products can readily be identified.

In this focus review, recent achievements of the author and his colleagues will be described. First, studies on molecular assemblies, in which the hydrogen bonding ${ }^{8}$ and halogen bonding have a role in the condensation, ${ }^{9}$ will be introduced. Second, mechanical properties of the conjugated polymers ${ }^{10,11}$ will be discussed. Then, synthesis and analysis of the doped graphene nanoribbon will be discussed. ${ }^{12}$

\section{SELF-ASSEMBLY VIA HYDROGEN BONDING}

To investigate intermolecular interaction in the nanoscale regime, studies of molecular assembly on surfaces have been extensively conducted with STM ${ }^{13-19}$ and $\mathrm{AFM}^{20-26}$ in the past two decades. The actual arrangement of the molecules is the result of a delicate balance between the molecule-molecule and molecule-substrate interactions. Among various intermolecular interactions, a hydrogen bonding is more interesting due to its directional interaction, ${ }^{27}$ compared with non-directional interactions such as van der Waals and electrostatic interactions. Here obtaining detailed structural information about supramolecular systems condensed by weak hydrogen bonding is discussed. ${ }^{8}$

Figure 1a shows the molecular structure of $\{[4-(\{4-[(2,3,4,5,6-$ pentafluorophenyl)ethynyl]-2,3,5,6-tetrafluorophenyl\}ethynyl)phenyl] ethynyl \}benzene (FFPB), ${ }^{28}$ in which four benzene rings are connected by ethynylene units. Among them, two benzene rings are substituted with fluorine. We expected that the strong dipole moment caused along the longitudinal axis $(4.27 \mathrm{D})$ can be used to condense the molecules when FFPB was deposited on a $\mathrm{Au}(110)-(1 \times 2)$ surface (Figure $1 \mathrm{~b}$ ) as forming one-dimensional structure along the $\left[\begin{array}{lll}1 & -1 & 1\end{array}\right]$ direction. However, the observed structure was formed along the $\left[\begin{array}{lll}2 & -2 & 3\end{array}\right]$ (Figure 1c). Furthermore, the width of the assembly was wider than that of the crosswise FFPB. Therefore, it was readily concluded that FFPB was not condensed by the strong dipole but rather by the weak $\mathrm{C}-\mathrm{H} \cdots \mathrm{F}$ hydrogen bonding. The corresponding high-resolution STM images, obtained with tip bias voltages of 1.6 and $-1.6 \mathrm{~V}$, were shown in Figures $1 \mathrm{~d}$ and e, respectively. In both images, only three maxima were observed in each FFPB although it has four benzene rings. To investigate the STM contrast, we assigned

${ }^{1}$ International Center for Materials Nanoarchitectonics, National Institute for Materials Science, Ibaraki, Japan and ${ }^{2}$ PRESTO, Japan Science and Technology Agency, Saitama, Japan

Correspondence: Dr S Kawai, International Center for Materials Nanoarchitectonics, National Institute for Materials Science, 1-1, Namiki, Tsukuba, Ibaraki 305-0044, Japan. E-mail:KAWAI.Shigeki@nims.go.jp

Received 1 July 2016; revised 28 September 2016; accepted 28 September 2016; published online 19 October 2016 
a<smiles>Fc1c(F)c(F)c(C#Cc2ccc(C#Cc3ccccc3)cc2)c(F)c1F</smiles>

b

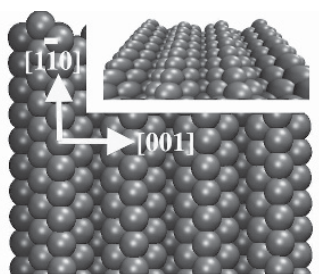

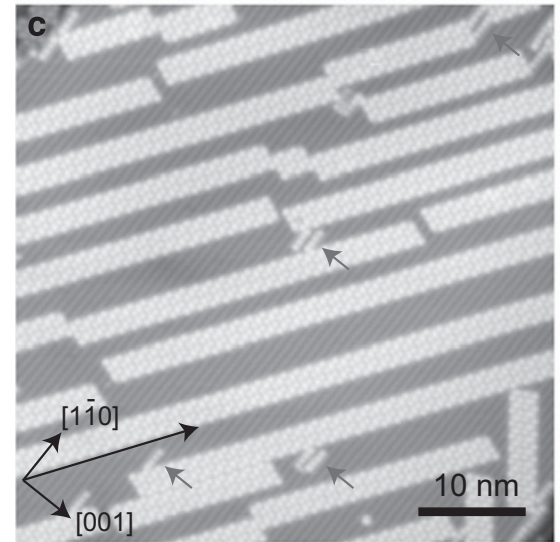

d
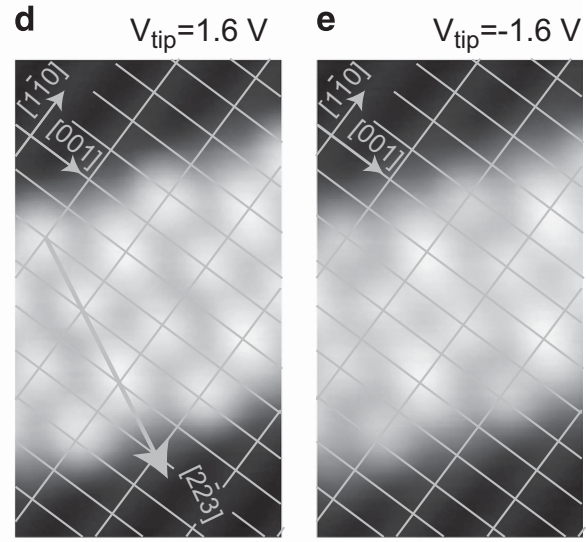

g

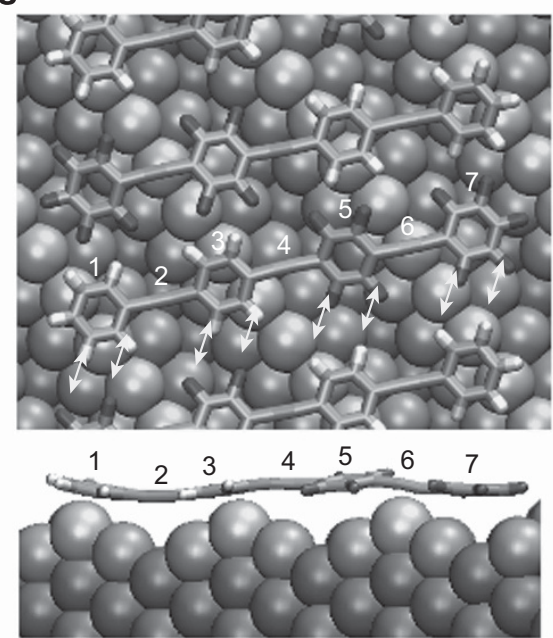

Figure 1 (a) Chemical structure of the FFPB molecule. (b) Top view of the Au(111)-(1×2) surface with atoms (inset: side view) (c) Large-area scanning tunneling microscopy topography of the self-assembly. (d, e) Close view of the molecules obtained with two different voltages. (f) High-resolution atomic force microscopy image obtained with a FFPB tip. (g) Top and side views of the relaxed molecule on the substrate using density functional theory calculations. A full color version of this figure is available at Polymer Journal online.

the lattice mesh of the substrate and found that the maxima in the STM images locate on top of the Au surface atoms. Apparently, instead of the molecular structure, the electronic properties of the substrate mainly affect to the observed contrasts.

In contrast to STM, AFM senses the total electron density. Especially, the tip starts to detect the short-range Pauli repulsive interaction at the small tip-sample separation. ${ }^{29}$ In this case, the detailed structure of molecules can be measured by detecting the frequency shift of the oscillating force sensor. ${ }^{4}$ However, before the tip reaches to the senses the Pauli repulsion region, a strong attractive metal tip-molecule interaction usually damages the assembly. To avoid this inadequate surface modification, the reactivity of the metal tip was reduced by terminating with a single FFPB. The so-called FFPB functionalized tip was in situ prepared by picking up from the surface and used for the high-resolution AFM imaging of the molecular self-assembly (Figure 1f). The inner structures of FFPB are clearly observed. As the tip is in the repulsive region at constant height, a convex appears as brighter contrast (lesser negative frequency shift). We found that the corrugation of $\mathrm{Au}(111)-(1 \times 2)$ substrate induces the rotation and/or bending of the benzene rings at the flexible single bonds in the acetylene moieties. To gain a better understanding, we conducted density functional theory calculations (Figure 1g). As the detailed structural information was obtained in the AFM image, only a few initial positions were taken. Consequently, we found that the detailed geometrical deformations of the molecule such as the rotation and tilt of the benzene rings as well as the bending of the single bonds are in agreement with the AFM image at atomic scale. In a detailed investigation, the sizes of the non- and fluoro-substituted benzenes in FFPB appear different. The difference can be enlarged up to $50 \%$ if the $\mathrm{CO}$ functionalized tip was used. With the state-of-the-art $a b$ initio 
calculation, it was found that the different extents of their $\pi$-electrons mainly relate to this feature. ${ }^{30}$

\section{SELF-ASSEMBLY VIA HALOGEN BONDING}

A halogen is intrinsically negatively charged. However, when it covalently bonds to another carbon atom in the molecule, an area of the positive electrostatic potential appear on the outermost portion of the halogen atom along the carbon-halogen $\sigma$ bond axis, the so-called $\sigma$-hole. ${ }^{31}$ This anisotropic distribution of the electrostatic potential is responsible for the halogen bond to a Lewis base. As the positive part is localized on the small cap, the halogen bond is more directional than the hydrogen bond. ${ }^{32}$ The polarizability of the halogen atom increases in the order of $\mathrm{Cl}, \mathrm{Br}$ and $\mathrm{I}$, and so that the strength of the positive caps as well as the halogen bonding increases in the same order. As the belt of the halogen atoms still has a negative electrostatic potential, the $\sigma$-hole can also bind to the belt of the halogen atom in an adjacent molecule, the so-called halogen $\cdots$ halogen bond. However, as fluorine has the strongest electronegativity and a low polarizability, it has no $\sigma$-hole in fluoro-substituted hydrocarbons such as $\mathrm{C}_{6} \mathrm{~F}_{6}$ and $\mathrm{CF}_{4}$. Therefore, we expected that fully fluoro-substituted phenyleneethynylene (1,2-bis\{2,3,5,6-tetrafluoro-4$[(2,3,4,5,6$-pentafluorophenyl)ethynyl $]$ phenyl $\}$ ethyne, ${ }^{28}$ BPEPE-F18, Figure 2a) cannot be condensed on a surface in the case of a submonolayer film.

Contrary to our expectations, BPEPE-F18 deposited on $\operatorname{Ag}(111)$ forms a well-ordered assembly (Figure 2b). ${ }^{9}$ The structure grew in the $[2-31]$ direction and its domain size was $>100 \times 100 \mathrm{~nm}$. The inset shows the close-up view, in which four protrusions correspond to the benzene rings. Among them, the two rings at the ends appear higher than the central two with an applied tip bias voltage of $-200 \mathrm{mV}$. To investigate the condensation mechanism, we obtained the detailed structural information with a CO functionalized tip of AFM. Figure 2c shows the high-resolution AFM image of the film, in which the molecule lies flat on $\mathrm{Ag}(111)$. As AFM image gives more geometrical information, the apparent height difference in the STM topography (Figure 2b) relates to the localized orbitals on the pentafluorobenzene moieties. To obtain a higher signal-to-noise ratio, the mesh-averaged filtering was applied over the unit cell. Figure $2 \mathrm{~d}$ shows the enlarged image in the area indicated by a square box in Figure $2 \mathrm{c}$. The hexagonal benzene rings and even the C-F bonds are clearly observed. As the effect of the CO tip tilt is sensitive enough to detect the bond order ${ }^{6}$ and the extended electronic cloud, ${ }^{30}$ it is not trivial to assign the bond length and angle directly from the observed image. Therefore, the positions of atomic cores were measured by assigning the chemical structure of BPEPE-F18 with the known dimension. As indicated with broken white lines (Figure 2e), each C-F bond points to a fluorine atom in the adjacent molecule, which results in highly directional C-F $\cdots$ F bonds $\left(\sim 180^{\circ}\right)$. Consequently, the angles between the C-F..F bonds are $\sim 120^{\circ}$. These features are the same as the halogen bonding. However, the measured length of the F $\cdots$ F gap is at least $300 \mathrm{pm}$, which is slightly larger than twice the van der Waals radius of fluorine (294 pm). This large gap is clear evidence that this bond differs from the typical halogen $\cdots$ halogen bonding.

To understand the mechanism of the directional C-F...F bonding, the electrostatic potentials (MEP) ${ }^{31}$ of BPEPE-F18 (Figure 2f) were calculated. Due to the high electronegativity of fluorine, the MEP on the outer shell stays negative as expected. In a detailed inspection, the MEP on the fluorine atom varies, resulting in the lesser negative value at the cap. However, as no positive part appears (no $\sigma$-hole), the electrostatic interaction in the F...F contact stays repulsive, which is consistent with the fact that the gap is larger than twice the van der
Waals radius of fluorine. Next, we performed extensive periodic calculations, including both BPEPE-F18 and $\mathrm{Ag}(111)$ substrate, and obtained the condensed conformation as observed in experiment. As the PBE functional together with empirical van der Waals correction was used in the calculation, we can estimate the contribution of the van der Waals attraction. This analysis allows us to find the condensation mechanism. The dispersion force takes the major part in the attraction, but the driving force of the directional bonding is due to the anisotropically distributed electrostatic potential around the fluorine atoms (that is, the lesser negative potential due to the C-F $\sigma$ bond). The presented C-F $\cdots$ F bonding has a high similarity to the typical halogen $\cdots$ halogen bonding in a sense of the directionality and influence of the anisotropically distributed electrostatic potential while the local interaction is repulsive.

\section{MECHANICAL PROPERTY MEASUREMENT OF A CONJUGATED MOLECULE}

$\mathrm{AFM}^{33}$ can be used not only for high-resolution imaging but also for force measurement. The so-called force spectroscopy has been applied to study the mechanical behavior of polymers ${ }^{34}$ and more complex biomolecules such as DNA complementary strands ${ }^{35}$ and protein, aiming to extend them by the applied force in a controlled manner. ${ }^{36}$ These measurements were mostly conducted in biological environment, meaning in solution at room temperature. Although these studies have the undisputed merit, little is known even about the mechanical behavior of single polymers pulled off a clean surface under the condition that both objects are priori identified on the atomic scale. A combination of high-resolution imaging and force spectroscopy by AFM at low temperature allows us to determine the energetic landscape of polymers interacting with a surface under controlled condition without any significant thermal fluctuations and drifts. ${ }^{10}$

In contrast to solution chemistry, it is extremely challenging to prepare an isolated, single and long molecular chain on an atomically clean surface under ultrahigh vacuum condition. Due to their large molecular weight, conventional thermal evaporation techniques cannot be used for the deposition of polymers without damaging. For this reason, it is mandatory to employ in situ polymerization technique of small precursor molecules on surfaces. In this study, we used dibromoterfluorene to synthesize polyfluorene using Ullmanntype on-surface chemical reaction on $\mathrm{Au}(111) .{ }^{37}$ Figure $3 \mathrm{a}$ shows the STM topography of the synthesized polyfluorene with a length of $20 \mathrm{~nm}$. As the number of molecular unit is 24 , eight precursors were conjugated. After the Au tip apex was positioned at the terminus of the polyfluorene as indicated by an arrow, a junction was established by setting the tip close enough to the terminus. When a strong junction between the Au tip and the last molecular unit is established, the abrupt change of the tip-sample interaction induces a large frequency shift of an oscillating force sensor. After the event, the tip was retracted in the vertical direction up to a distance of $25 \mathrm{~nm}$. Figure $3 \mathrm{~b}$ shows the recorded distance dependence of the frequency shift. We calculated the effective stiffness as $k_{\mathrm{ts}}=-2 k_{\mathrm{c}} \Delta f / f$, where $k_{\mathrm{c}}$ is the stiffness of the force sensor, $f$ the resonance frequency and $\Delta f$ the measured frequency shift and indicated the corresponding scale on the right. ${ }^{38,39}$ A periodic variation of the frequency shift is observed clearly, in which each period was ended by an abrupt shift as indicated by broken lines. In contrast to previous conductance measurements, ${ }^{37}$ the magnitude of the measured frequency shift modulation almost stays constant, whereas the length of the free-standing chain segment increases. Therefore, we can readily study the mechanical behavior of the polymer until complete pull off from the surface. In Figure 3b, we 
a<smiles>Fc1c(F)c(F)c(C#Cc2c(F)c(F)c(C#Cc3c(F)c(F)c(C#Cc4c(F)c(F)c(F)c(F)c4F)c(F)c3F)c(F)c2F)c(F)c1F</smiles>
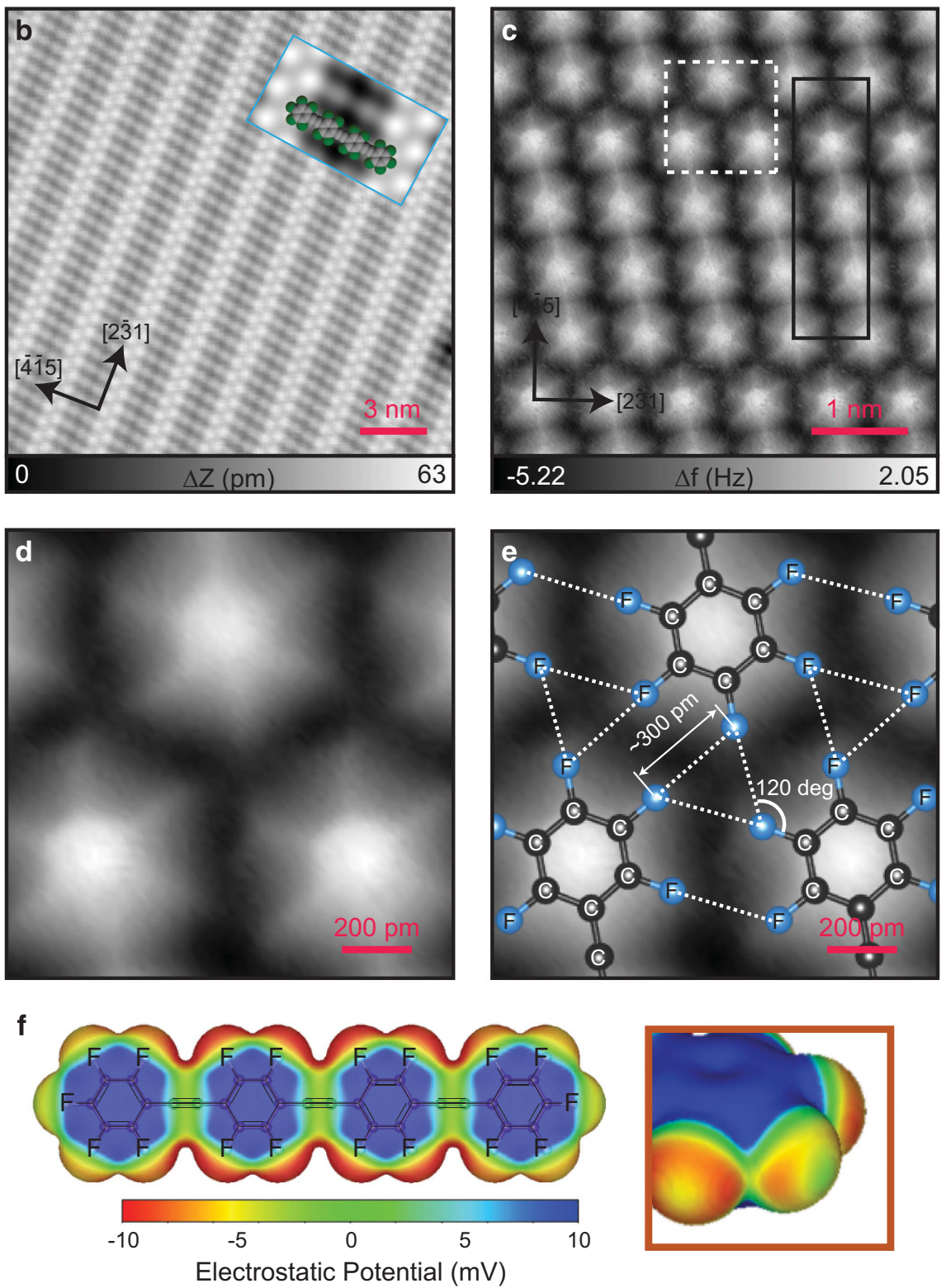

Figure 2 (a) Chemical structure of BPEPE-F18. (b) Large-area STM topography of the self-assembly. (c) High-resolution AFM imaged obtained with a CO tip. A black rectangle shows the unit cell. (d) Magnified AFM image, indicated by a white area in c. (e) Same as d with a superimposed stick-and-ball drawing of BPEPE-F18. (f) Calculated molecular electrostatic potentials of BPEPE-F18 projected on the constant density $\left(0.001\right.$ e/Bohr $\left.^{3}\right)$ surface.

observe 24 periods of the frequency shift modulation, corresponding to the initial number of molecular units on $\mathrm{Au}(111)$. Furthermore, the mean distance between adjacent jumps $(0.91 \pm 0.07 \mathrm{~nm})$ is close to the length of a fluorene unit $(0.845 \mathrm{~nm})$ and also stays constant for any length. Therefore, the polyfluorene was stepwise detached from the substrate unit by unit, whereas the units remained on the surface slid laterally. In other words, the detachment site of the molecular units stayed on the surface almost under the tip apex, moving in the vertical direction. The frequency shift suddenly jumps to zero at $Z=21 \mathrm{~nm}$, that is, at a distance close to the length of the polymer on the surface. The complete detachment was also confirmed by the absence of the chain in the STM topography recorded after the pulling measurement (Figure 3a). In a closer inspection of a single detachment event (Figure 3c), there are minor modulations, whose periodicity is close to the value of the Au lattice spacing along an fcc stripe $(0.5 \mathrm{~nm})$.

To investigate the mechanisms inducing the periodicity shift in the pulling process of the polyfluorene, model calculations were performed. Following an idea of the Frenkel-Kontorova model, ${ }^{40}$ 
a

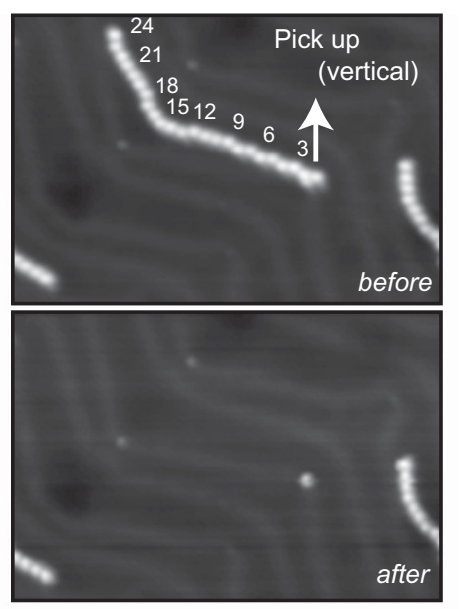

b
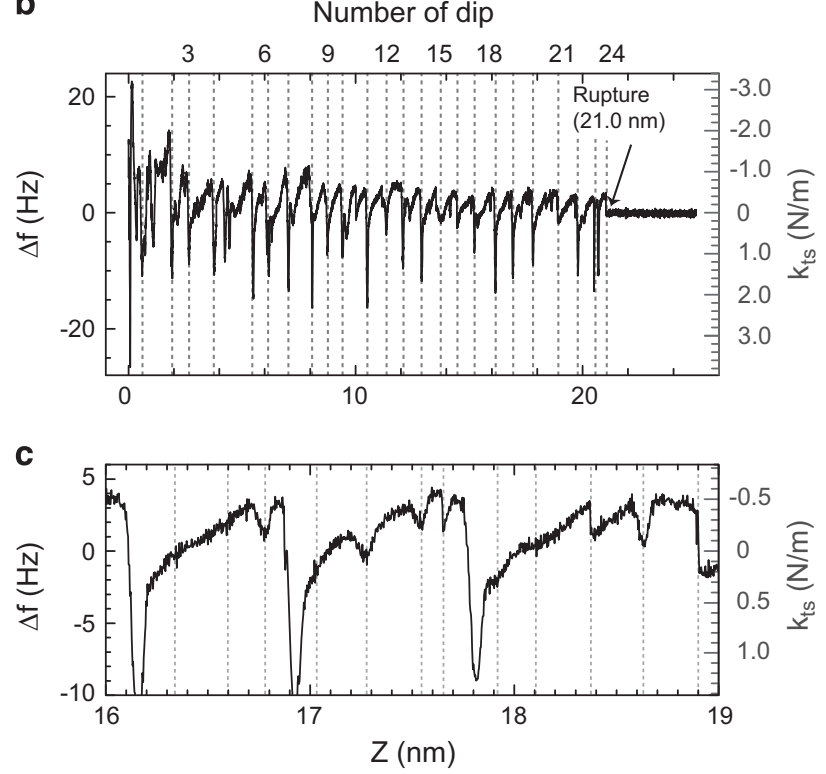
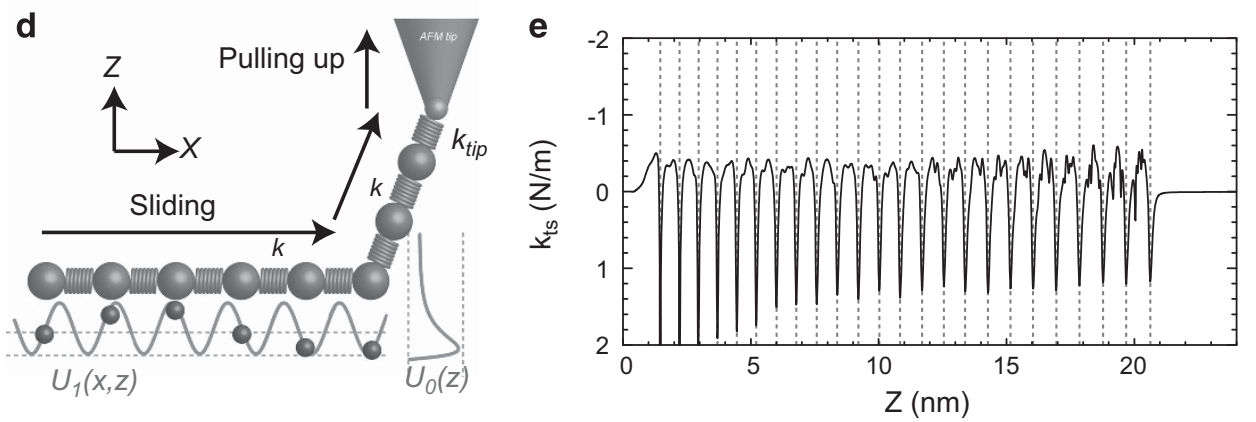

Figure 3 (a) STM topographies of the same surface area before and after pulling off a single polyfluorene initially lying along an fcc stripe of the herringbones reconstruction of $\mathrm{Au}(111)$. (b) Recorded frequency shift of an oscillating force sensor while retracting the tip after contacting. The detachment event was indicated by an arrow. (c) Magnification around $Z=17.5 \mathrm{~nm}$. (d) Schematic representation of the interactions acting while pulling up the polymer. (e) Calculated $Z$ distance dependence of the normal force gradient. A full color version of this figure is available at Polymer Journal online.

the units of polyfluorene were regarded as particles, harmonically coupled each other and adsorbed on the surface with realistic adsorption $U_{0}$ and corrugation potentials $U_{1}$ (Figure $3 \mathrm{~d}$ ). ${ }^{41}$ Figure $3 \mathrm{e}$ shows the representative results, in which the sequential detachment of the units with a period of close to the unit length is the most prominent feature as seen in experiment (Figure 3b). Besides the main signal, a small modulation related to the $\mathrm{Au}(111)$ substrate lattice is also represented. As this small modulation is superimposed on the main ones, it does not appear periodic. Such variation is related to the misfit between the $\mathrm{Au}(111)$ lattice distance and the unit length. Our model calculation provided important information. The adsorbed portion of polyfluorene slides very easily on $\mathrm{Au}(111)$ during the pulling process, inducing only small modulations of the frequency shift. This friction behavior relates to the high lateral stiffness of the molecular unit and their length mismatch with respect to the substrate lattice, namely, incommensurability. Otherwise, the polymer cannot be slid by the tip-induced manipulation due to high friction.

\section{FRICTION OF A POLYMER ON A SURFACE}

In the previous section, we discussed that the incommensurability between the molecular unit and the substrate has a key role in friction. ${ }^{10}$ Polyfluorene is consisted of units, which are connected via a single bond. The bond can be angled and rotated with a relatively low-energy cost as each unit tends to adsorb on a stable atomic site on the substrate. Therefore, the incommensurability is in fact moderate. To get a contact with an extremely high incommensurability, that is, super-lower friction, a high lateral stiffness of the sliding object is required. Graphene offers unique properties as a solid lubricant. ${ }^{42}$ The interpretation of such superlubric behavior is based on the hypothesis that the high lateral stiffness as well as the weak interaction with most materials makes an incommensurable contact with most solid surfaces. ${ }^{43}$ To substantiate this promise and establish a connection with the tribological properties observed on macro- and mesoscales, it is highly desirable to measure the frictional property of a graphene nano-flake, such as graphene nanoribbon (GNR), ${ }^{11}$ on an atomically well-defined surface.

GNRs recently have been synthesized on metal surfaces from anthracene derivatives by an on-surface chemical reaction. ${ }^{44}$ Using AFM, we investigated the mechanical response of GNRs with the tip-induced lateral manipulation. This study originated from the unintentional manipulation of GNRs aligned along the $\left[\begin{array}{lll}-1 & 0 & 1\end{array}\right]$ direction of the $\mathrm{Au}(111)$ surface, when the STM topography was taken with a $\mathrm{Au}$ tip (Figure 4a) even at low temperature. Although a relatively large tip-sample separation was set, the GNRs were always moved along their longitudinal axis, indicating high diffusivity. To measure the static friction, we switched to AFM and acquired a 

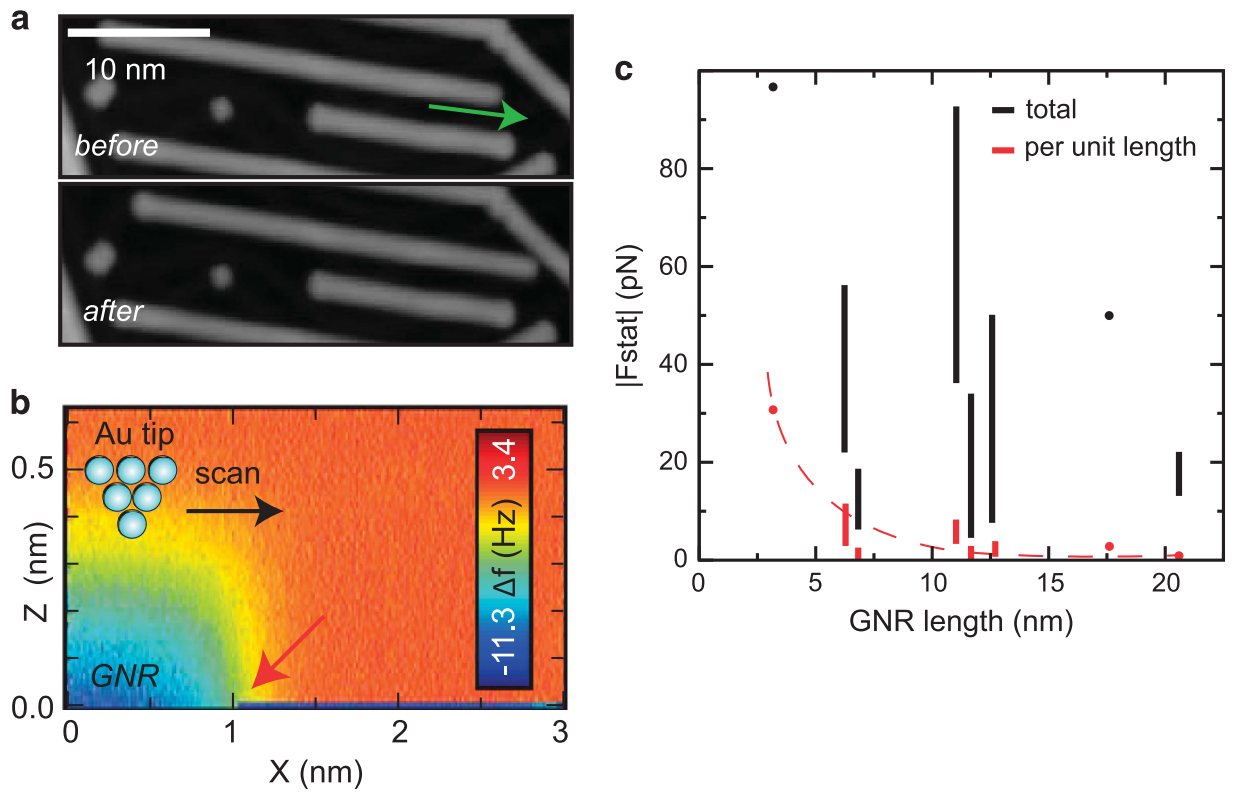

Figure 4 (a) STM topographies of graphene nanoribbons (GNRs) on Au(111) before and after a tip-induced lateral manipulation. (b) Two-dimensional frequency shift map along the longitudinal axis of the manipulated GNR. (c) The absolute value of $F_{\text {stat }}$ as a function of the GNR length (black) and $F_{\text {stat }}$ per unit length (red).

two-dimensional frequency shift map around the edge, whereas the Au tip was scanned laterally along the longitudinal axis of the GNR at different constant $Z$ heights. Following the method proposed by Ternes et al. ${ }^{45}$ the tip-GNR separation was reduced stepwise until an abrupt frequency shift was detected (Figure 4b). To quantify the static force, the energy landscape was first calculated by integrating two times the frequency shift along the $Z$ direction and was then differentiated along the $X$ direction. It was found that the manipulation occurred with a static force of $-105 \mathrm{pN}$. The magnitude of the force is exceptionally low, considering that the number of atoms of GNR is much larger than that of the previously manipulated single atoms ${ }^{45}$ and conventional molecules. ${ }^{46}$ Our result indicates the superlubric properties of GNRs. Another signature of superlubricity is the fact that the static friction force per unit of contact area decreases with increasing the length of GNR. To investigate this, the static friction of GNRs on $\mathrm{Au}(111)$ with different lengths was measured (Figure 4c). Although the measured data spread due to influence of the reconstructed surface, we found that the force per unit length decreases with increasing the GNR length.

To gain a detailed insight into the dynamics of the sliding motion of GNR, we applied the procedure introduced in the previous section and succeeded in attaching a short zig-zag edge of a GNR to a Au-coated tip (Figure 5a). Here we laterally moved the GNR forward and backward along the $\left[\begin{array}{lll}-1 & 0 & 1\end{array}\right]$ direction of $\mathrm{Au}(111)$ at different constant $Z$ heights (Figure $5 \mathrm{~b}$ ). Figures $5 \mathrm{c}-\mathrm{f}$ show the corresponding variations of the frequency shift. We found that the frequency shift oscillated with a periodicity of $0.28 \mathrm{~nm}$, except when the GNR was moved backward at $Z=5 \mathrm{~nm}$. The amplitude of the frequency shift modulations varies along $X$ and was changed by a factor of 2 . We also observed curves with roughly half-periodicity at a small scanning distance of $z=1$ to $2 \mathrm{~nm}$. The corresponding molecular dynamics simulation reproduced the experimental observations. We found that the frequency shift curve is significantly modulated by the herringbone reconstruction, which is caused by the deformations in the top $\mathrm{Au}(111)$ layer. Although the corresponding corrugation amplitude is tiny $(20 \mathrm{pm})$, the commensurability degree is significantly modulated, leading the same modulation in friction. As the short zig-zag edge binds more strongly to the substrate than to the inner atoms, the friction force per unit length decreases with increasing the GNR length as observed in the experiment. Our molecular dynamic simulations are consistent with the commonly accepted interpretation of the structural superlubricity of graphene. The detailed dynamics of the sliding motion are nevertheless affected by local surface properties as the surface reconstruction, relating to the variable degree of commensurability.

\section{SYNTHESIS OF BORON-DOPED GRAPHENE NANORIBBON}

Doping another element (that is, boron and nitrogen) to GNRs reduces the incommensurability so that the friction increases. Besides the interest in the mechanical behavior, electron deficiency and Lewis acidity of boron are essential to tune the properties of the GNR and graphene. Incorporation of boron atoms into graphene offers a wide variety of functionality in chemical sensing, ${ }^{47}$ nanoelectronics, ${ }^{48}$ photocatalysis, ${ }^{49}$ battery electrodes ${ }^{50}$ and so on. However, the doping site and density of boron in graphene have never been precisely controlled. In this section, synthesis of boron-doped graphene nanoribbons (B-GNRs) of widths of $N=7,14$ and 21 are described. ${ }^{12}$

To dope boron atoms in GNRs, the precursor (9,10-bis (10-bromoanthracen-9-yl)-9,10-dihydro-9,10-diboraanthracene) was designed and synthesized (Figure 6a). The molecule is composed of two pristine anthracenes and one 9,10-dibora-9,10-dihydroanthracene, covalently bonded each other. Furthermore, both termini are substituted by bromine atoms. Annealing the precursor molecules on $\mathrm{Au}(111)$ at $180^{\circ} \mathrm{C}$ induce the surface catalytic debromination and then the polymerization of the precursor molecules. At an elevated temperature of up to $400^{\circ} \mathrm{C}$, an aromatic B-GNR is formed by the surface-assisted cyclodehydrogenation ${ }^{51}$ (Figure 6a), similar to the pristine GNR. ${ }^{44}$ Figure $6 \mathrm{~b}$ shows the STM image of B-GNRs after annealing at $400{ }^{\circ} \mathrm{C}$. In a close view, the STM topography of the 
a

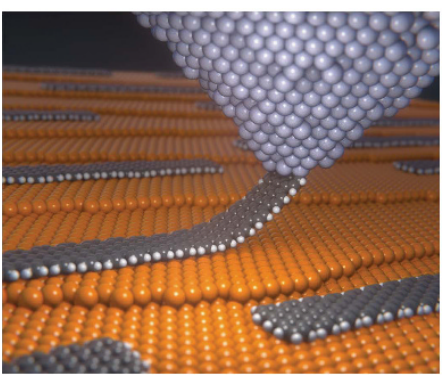

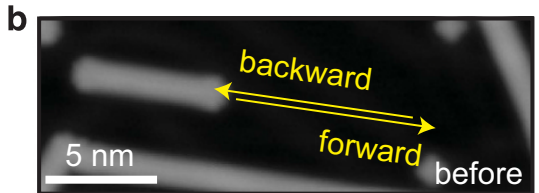

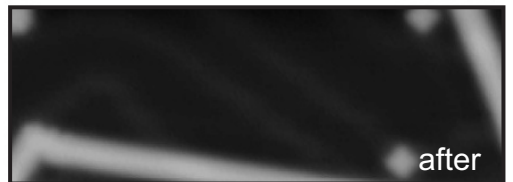

c

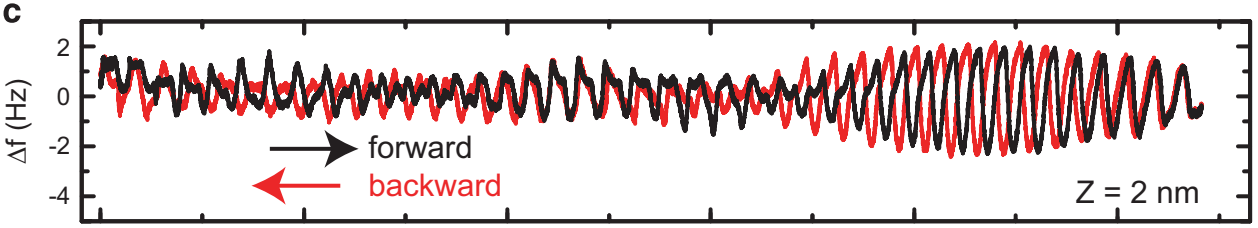

d
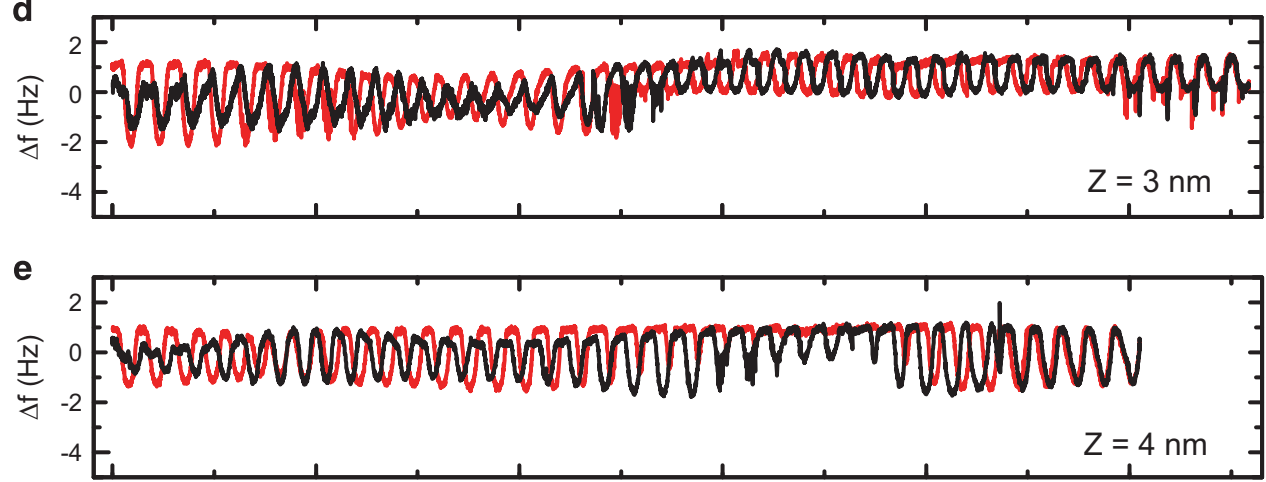

f

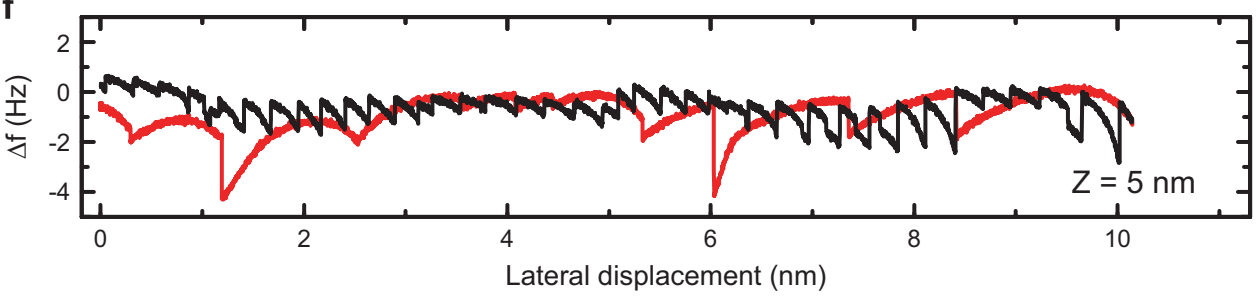

Figure 5 (a) Schematic drawing of the lateral manipulation of GNR. (b) STM topographies before and after the GNR has been displaced by a Au tip in the directions indicated by yellow arrows. (c-f) Frequency shifts of an oscillating forces sensor accompanying the lateral motion at different $Z$ heights.

B-GNR is inverted by changing the applied tip bias voltage of $-3 \mathrm{~V}$ (Figure 6c) to $+3 \mathrm{~V}$ (Figure $6 \mathrm{~d}$ ), indicating the perturbation of the electronic structure due to the presence of the doped boron as an electron-accepting element. The pink crosses indicate the same atomic site in B-GNR. By setting close to zero bias voltage ( $-2 \mathrm{mv})$, the electronic states near the Fermi level can be detected (Figure 6e). The ratio of one dark and two bright contrasts at the armchair type edge indicates that the dark and bright contrast edges relate to the boron-doped and pristine anthracenes, respectively. More detailed structural information was obtained by scanning with a $\mathrm{CO}$ functionalized tip of AFM in a constant $Z$ height mode. Figure $6 \mathrm{f}$ shows the corresponding high-resolution AFM image of B-GNR, in which a defect-like feature was seen in every three anthracene units. After applying a Laplace filter to the observed AFM image, the C-C bonds and even the $\mathrm{B}-\mathrm{C}$ bonds appear clearly (Figure $6 \mathrm{~g}$ ). To investigate the mechanism of the dark and faint contrast around the boron site, we performed density functional theory calculations and found that the boron atom locates closer to the $\mathrm{Au}(111)$ substrate by $30 \mathrm{pm}$. This topographic corrugation has an important role in the frequency shift in a constant height mode imaging (Figure 6h). After the substrate was annealed at higher temperature of $510^{\circ} \mathrm{C}$, B-GNRs were fused at the armchair edges. Consequently, B-GNRs with wider widths $(N=14$ and 21$)$ were synthesized (Figure 6i). For $N=14$ B-GNRs, two different fused configurations with the out-of-phase (Figure 6j) and the shifted phase (Figure 6k) were observed. As the configuration of $N=14 \mathrm{~B}-\mathrm{GNR}$ is determined by the relative position in the fusing process, the shifted phase one has a mirror configuration. Therefore, three textures exist in total for $N=14$ B-GNR and nine for $N=21$ B-GNRs. The perfectly order out-ofphase structure is shows in figure 6l. More interestingly, the boron atoms can stay at the designed atomic site, although it was heated up to $510{ }^{\circ} \mathrm{C}$ during the fusing process. Therefore, once a boron atom in perfectly incorporated in graphene, the thermal stability of the $\mathrm{BC}_{3}$ site is remarkably high. 

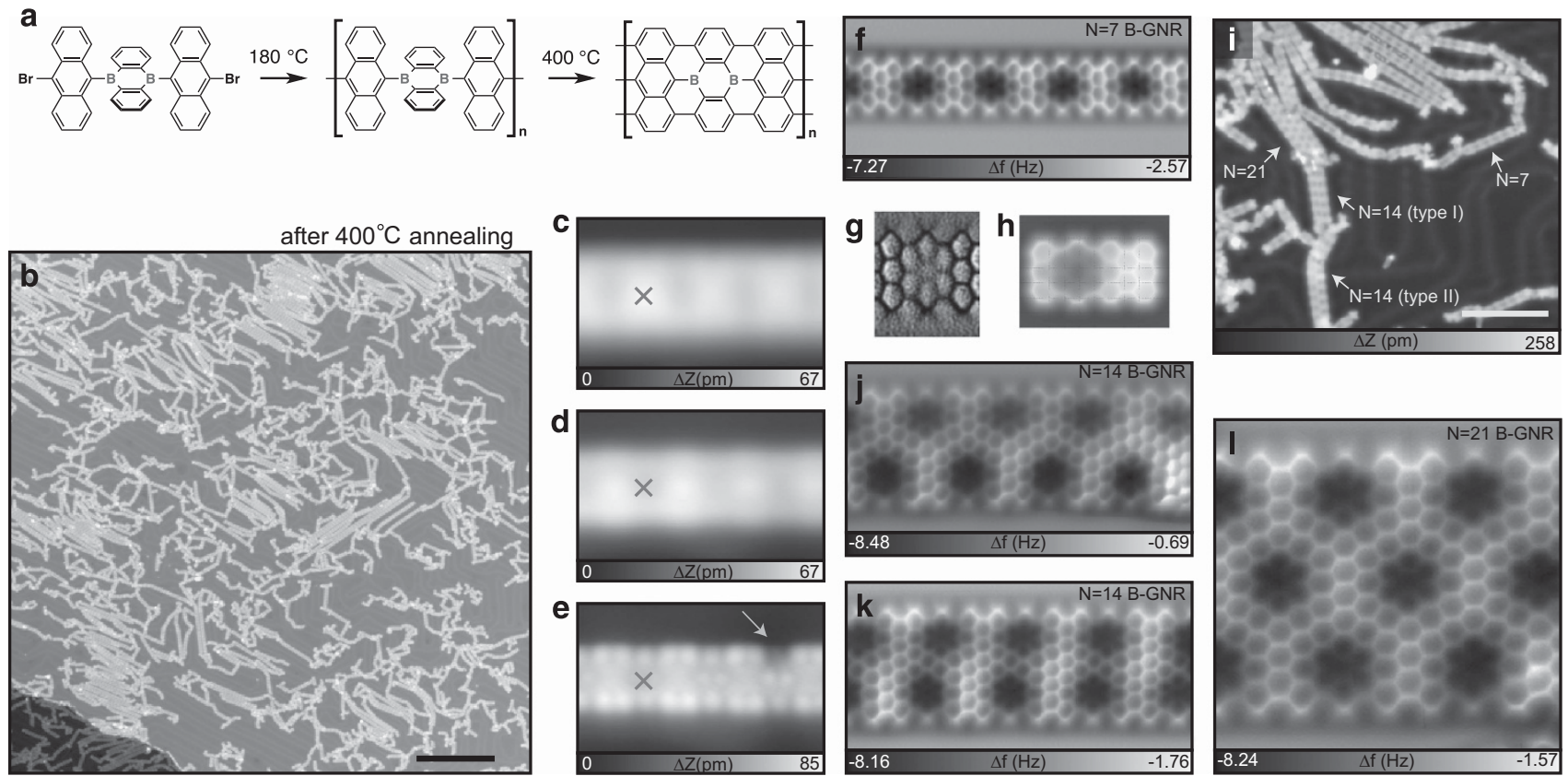

Figure 6 (a) Schematic drawing of the on-surface chemical reaction for B-GNR. (b) STM topography of B-GNR, synthesized on Au(111). Scale bar, 30 nm. (c-e) High-resolution STM topographies taken with tip bias voltages of $-3 \mathrm{~V}, 3 \mathrm{~V}$ and $-2 \mathrm{mV}$. (f) High-resolution AFM image of $N=7 \mathrm{~B}-\mathrm{GNR}$ and (g) the corresponding Laplace filtered image for a better view of bonds. (h) Simulated AFM image. (i) STM topography after annealing at $510{ }^{\circ} \mathrm{C}$. Scale bar, $10 \mathrm{~nm}$. (j, k) AFM images of $N=14$ B-GNR and (I) of $N=21$ B-GNR. A full color version of this figure is available at Polymer Journal online.

\section{CONCLUSION}

In this focus review, recent developments in the field of AFM for onsurface chemistry were described. A direct observation of inner structures of molecules is beneficial to study single and selfassembled molecules as well as chemical reactions on the surface. Especially, on-surface chemical reaction can synthesize polymers on an atomically clean surface and so that the electronic ${ }^{37}$ and mechanical properties $^{10,11}$ can be in-depth studied. Besides such fundamental studies, a novel application, that is, electroluminescence, ${ }^{52}$ can be foreseen.

\section{CONFLICT OF INTEREST}

The author declares no conflict of interest.

\section{ACKNOWLEDGEMENTS}

This work was supported in part by the Japan Science and Technology Agency (JST) 'Precursory Research for Embryonic Science and Technology (PRESTO)' for a project of 'Molecular technology and creation of new function', by JSPS KAKENHI grant number 15K21765. I thank E Meyer, A Baratoff, AS Foster, A Sadeghi, A Benassi, R Fasel, X Feng, T Glatzel, E Gnecco, S Goedecker, L Grill, L Gross, S Hecht, M Koch, G Meyer, N Moll, K Müllen, A Orita, S Osumi, J Otera, D Passrone, R Pawlak, L Peng, CA Pignedoli, P Ruffieux, S Saito, B Schuler, J Schwarz, H Söde, P Spiker, A Willand, F Xu and $S$ Yamaguchi for the valuable discussions and collaborative research activities, described in this focus review article.

1 Binnig, G., Rohrer, H., Gerber, C. \& Weibel, E. Surface studies by scanning tunneling microscopy. Phys. Rev. Lett. 49, 57-61 (1982).

2 Barth, J. V., Costantini, G \& Kern, K. Engineering atomic and molecular nanostructures at surfaces. Nature 437, 671-679 (2005).

3 Repp, J., Meyer, G., Stojković, S. M., Gourdon, A. \& Joachi, C. Molecules on insulating films: scanning-tunneling microscopy imaging of individual molecular orbitals. Phys. Rev. Lett. 94, 026803 (2005).
4 Gross, L., Mohn, F., Moll, N., Liljeroth, P. \& Meyer, G. The chemical structure of a molecule resolved by atomic force microscopy. Science 325, 1110-1114 (2009).

5 Moll, N., Gross, L., Mohn, F., Curioni, A. \& Meyer, G. The mechanisms underlying the enhanced resolution of atomic force microscopy with functionalized tips. New J. Phys. 12, 125020 (2010).

6 Gross, L., Mohn, F., Moll, N., Schuler, B., Criado, A., Guitián, E., Peña, D., Gourdon, A. \& Meyer, G. Bond-order discrimination by atomic force microscopy. Science 337, 1326-1329 (2012).

7 de Oteyza, D. G., Gorman, P., Chen, Y.-C., Wickenburg, S., Riss, A., Mowbray, D. J., Etkin, G., Pedramrazi, Z., Tsai, H.-Z., Rubio, A., Crommie, M. F. \& Fischer, F. R. Direct imaging of covalent bond structure in single-molecule chemical reactions. Science $\mathbf{3 4 0}$, 1434-1437 (2013)

8 Kawai, S., Sadeghi, A., Feng, X., Lifen, P., Pawlak, R., Glatzel, T., Willand, A., Orita, A., Otera, J., Goedecker, S. \& Meyer, E. Obtaining detailed structural information about supramolecular systems on surfaces by combining high-resolution force microscopy with ab initio calculations. ACS Nano 7, 9098-9105 (2013).

9 Kawai, S., Sadeghi, A., Xu, F., Peng, L., Orita, A., Otera, J., Goedecker, S. \& Meyer, E. Extended halogen bonding between fully fluorinated aromatic molecules. ACS Nano $\mathbf{9}$, 2574-2583 (2015)

10 Kawai, S., Koch, M., Gnecco, E., Sadeghi, A., Pawlak, R., Glatzel, T., Schwarz, J., Goedecker, S., Hecht, S., Baratoff, A., Grill, L. \& Meyer, E. Quantifying the atomic-level mechanics of single long physisorbed molecular chains. Proc. Natl Acad. Sci. USA 111, 3968-3972 (2014).

11 Kawai, S., Benassi, A., Gnecco, E., Söde, H., Pawlak, R., Feng, X., Müllen, K., Passerone, D., Pignedoli, C. A., Ruffieux, P., Fasel, R. \& Meyer, E. Superlubricity of graphene nanoribbons on gold surfaces. Science 351, 957-961 (2016).

12 Kawai, S., Saito, S., Osumi, S., Yamaguchi, S., Foster, A. S., Spijker, P. \& Meyer, E. Atomically-controlled substitutional boron-doping of graphene nanoribbons. Nat. Commun. 6, 8098 (2015).

13 Lorenzo, M. O., Baddeley, C. J., Muryn, C. \& Raval, R. Extended Surface Chirality from supramolecular assemblies of adsorbed chiral molecules. Nature 404, 376-379 (2000).

14 Yokoyama, T., Yokoyama, S., Kamikado, T., Okuno, Y. \& Mashiko, S. Selective assembly on a surface of supramolecular aggregates with controlled size and shape. Nature 413, 619-621 (2001).

15 Kühnle, A., Linderoth, T. R., Hammer, B. \& Besenbacher, F. Chiral Recognition in dimerization of adsorbed cysteine observed by scanning tunnelling microscopy. Nature 415, 891-893 (2002).

16 Theobald, J. A., Oxtoby, N. S., Phillips, M. A., Champness, N. R. \& Beton, P. H. Controlling molecular deposition and layer structure with supramolecular surface assemblies. Nature 424, 1029-1031 (2003).

17 Fasel, R., Parschau, M. \& Ernst, K.-H. Amplification of chirality in two-dimensional enantiomorphous lattices. Nature 439, 449-452 (2006).

18 Madueno, R., Räisänen, M. T., Silien, C. \& Buck, M. Functionalizing hydrogen-bonded surface networks with self-assembled monolayers. Nature 454, 618-621 (2008). 
19 Blunt, M. O., Russell, J. C., del Carmen Giménez-López, M., Garrahan, J. P., Lin, X., Schröder, M., Champness, N. R. \& Beton, P. H. Random tiling and topological defects in a two-dimensional molecular network. Science 322, 1077-1081 (2008).

20 Loppacher, C., Guggisberg, M., Pfeiffer, O., Meyer, E., Bammerlin, M., Lüthi, R. Schlittler, R., Gimzewski, J. K., Tang, H. \& Joachim, C. Direct determination of the energy required to operate a single molecule switch. Phys. Rev. Lett. 90, 066107 (2003)

21 Nony, L., Gnecco, E., Alkauskas, A., Baratoff, A., Bennewitz, R., Pfeiffer, O., Maier, S., Wetzel, A., E. Meyer, E. \& Gerber, C. Observation of individual molecules trapped on a nanostructured insulator. Nano Lett. 44, 2185-2189 (2004).

22 Maier, S., Fendt, L., Zimmerli, L., Glatzel, T., Pfeiffer, O., F. Diederich, F. \& Meyer, E. Nanoscale engineering of molecular porphyrin wires on insulating surfaces. Small 4, 1115-1118 (2008).

23 Such, B., Trevethan, T., Glatzel, T., Kawai, S., Zimmerli, L., Meyer, E., Shluger, A. L., Amijs, C. H. M., de Mendoza, P. \& Echavarren, A. M. Functionalized truxenes: Adsorption and diffusion of single molecules on the $\operatorname{KBr}(001)$ surface. ACS Nano 4, 3429-3439 (2010).

24 Kittelmann, M., Rahe, P., Nimmrich, M., Hauke, C. M., Gourdon, A. \& Kühnle, A. On-surface covalent linking of organic building blocks on a bulk insulator. ACS Nano 5, 8420-8425 (2011).

25 Rahe, P., Kittelmann, M., Neff, J. L., Nimmrich, M., Reichling, M., Maass, P. \& Kühnle, A. Tuning molecular self-assembly on bulk insulator surfaces by anchoring of the organic building blocks. Adv. Mater. 25, 3948-3956 (2013).

26 Lindner, R., Rahe, P., Kittelmann, M., Gourdon, A., Bechstein, R. \& Kühnle, A. Substrate templating guides photo-induced reaction of C60 on calcite. Angew. Chem. Int. Ed. 53, 7952-7955 (2014).

27 Sherrington, D. C. \& Taskinen, K. A. Self-Assembly in Synthetic Macromolecular systems via multiple hydrogen bonding interactions. Chem. Soc. Rev. 30, 83-93 (2001).

28 Matsuo, D., Yang, X., Hamada, A., Morimoto, K., Kato, T., Yahiro, M., Adachi, C., Orita, A. \& Otera, J. Fluoro-substituted phenyleneethynylenes: Acetylenic n-type organic semiconductors. Chem. Lett. 39, 1300-1302 (2010).

29 Moll, N., Gross, L., Mohn, F., Curioni, A. \& Meyer, G. A simple model of molecular imaging with noncontact atomic force microscopy. New J. Phys. 14, 083023 (2012).

30 Moll, N., Schuler, B., Kawai, S., Xu, F., Peng, L., Orita, A., Otera, J., Curioni, A., Neu, M., Repp, J., Meyer, G. \& Gross, L. Image distortions of a partially fluorinated hydrocarbon molecule in atomic force microscopy with carbon monoxide terminated Tips. Nano Lett. 14, 6127-6131 (2014).

31 Clark, T., Hennemann, M., Murray, J. S. \& Politzer, P. Halogen bonding: the $\sigma$-hole J. Mol. Model 13, 291-296 (2007).

32 Voth, A. R., Khuu, P., Oishi, K. \& Ho, P. S. Halogen bonds as orthogonal molecular interactions to hydrogen bonds. Nat. Chem. 1, 74-79 (2009).

33 Binnig, G., Quate, C. F. \& Gerber, C. Atomic force microscope. Phys. Rev. Lett. 56, 930-933 (1986).

34 Rief, M., Fernandez, J. M. \& Gaub, H. E. Elastically coupled two-level systems as a model for biopolymer extensibility. Phys. Rev. Lett. 81, 4764-4767 (1998).
35 Lee, G. U., Chrisey, L. A. \& Colton, R. J. Direct measurement of the forces between complementary strands of DNA. Science 266, 771-773 (1994).

36 Schlierf, M., Li, H. \& Fernandez, J. M. The unfolding kinetics of ubiquitin captured with single-molecule force-clamp techniques. Proc. Natl Acad. Sci. USA 101, 7299-7304 (2004).

37 Lafferentz, L., Ample, F., Yu, H., Hecht, S., Joachim, C. \& Grill, L. Conductance of a single conjugated polymer as a continuous function of its length. Science 323 1193-1197 (2009).

38 Albrecht, T. R., Grütter, P., Horne, D. \& Rugar, D. Frequency modulation detection using high- $Q$ cantilevers for enhanced force microscope sensitivity. J. Appl. Phys. 69 , 668-673 (1991).

39 Giessibl, F. J. Advances in atomic force microscopy. Rev. Mod. Phys. 75, 949-983 (2003).

40 Braun, O. \& Naumovets, A. Nanotribology. Microscopic mechanisms of friction. Surf Sci. Rep. 60, 79-158 (2006).

41 Steele, W. A. The physical interaction of gases with crystalline solids. Surf. Sci. $\mathbf{3 6}$, 317-352 (1973).

42 Berman, D., Erdemir, A. \& Sumant, A. V. Graphene: a new emerging lubricant. Mater. Today 17, 31-42 (2014).

43 Verhoeven, G. S., Dienwiebel, M. \& Frenken, J. W. M. Model calculations of superlubricity of graphite. Phys. Rev. B 70, 165418 (2004).

44 Cai, J., Ruffieux, P., Jaafar, R., Bieri, M., Braun, T., Blankenburg, S., Muoth, M., Seitsonen, A. P., Saleh, M., Feng, X., Müllen, K. \& Fasel, R. Atomically precise bottomup fabrication of graphene nanoribbons. Nature 466, 470-473 (2010).

45 Ternes, M., Lutz, C. P., Hirjibehedin, C. F., Giessibl, F. J. \& Heinrich, A. J. The force needed to move an atom on a surface. Science 319, 1066-1069 (2008).

46 Langewisch, G., Falter, J., Schirmeisen, A. \& Fuchs, H. Long jumps of an organic molecule induced by atomic force microscopy manipulation. Adv. Mater. Interfaces 1, 1300013 (2014).

47 Zhang, Y., Zhang, D. \& Liu, C. Novel chemical sensor for cyanides: boron-doped carbon nanotubes. J. Phys. Chem. B 110, 4671-4674 (2006).

48 Terrones, H., Mauricio Terrones, R. L. \& Dresselhaus, M. S. The role of defects and doping in 2D graphene sheets and 1D nanoribbons. Rep. Prog. Phys. 75, 062501 (2012).

49 Xing, M., Fang, W., Yang, X., Tian, B. \& Zhang, J. Highly-dispersed boron-doped graphene nanoribbons with enhanced conductibility and photocatalysis. Chem. Commun. 50, 6637-6640 (2014).

$50 \mathrm{Wu}$, Z.-S., Ren, W., Xu, L., Li, F. \& Cheng, H.-M. Doped graphene sheets as anode materials with superhigh rate and large capacity for lithium ion batteries. ACS Nano 5 , 5463-5471 (2011).

51 Treier, M., Pignedoli, C. A., Laino, T., Rieger, R., Müllen, K., Passerone, D. \& Fasel, R. Surface-assisted cyclodehydrogenation provides a synthetic route towards easily processable and chemically tailored nanographenes. Nat. Chem. 3, 61-67 (2011).

52 Reecht, G., Scheurer, F., Speisser, V., Dappe, Y. J., Mathevet, F. \& Schull, G. Electroluminescence of a polythiophene molecular wire suspended between a metallic surface and the tip of a scanning tunneling microscope. Phys. Rev. Lett. 112, 047403 (2014). 